\title{
Germanica
}

\section{Émotion et évaluation : le cas des adjectifs affectifs dans les critiques de Marcel Reich-Ranicki}

Dominique DIAS: Emotion und Bewertung: der Fall der emotionalen Adjektive in den Rezensionen von Marcel Reich-Ranicki

Dominique DIAS: Emotion and evaluation: the case of emotional adjectives in the reviews of Marcel Reich-Ranicki

\section{Dominique Dias}

\section{OpenEdition Journals}

Édition électronique

URL : https://journals.openedition.org/germanica/7902

DOI : 10.4000/germanica.7902

ISSN : 2107-0784

\section{Éditeur}

Université de Lille

\section{Édition imprimée}

Date de publication : 1 décembre 2019

Pagination : 97-110

ISBN : 978-2-913857-44-5

ISSN : 0984-2632

\section{Référence électronique}

Dominique Dias, «Émotion et évaluation : le cas des adjectifs affectifs dans les critiques de Marcel Reich-Ranicki », Germanica [En ligne], 65 | 2019, mis en ligne le 01 janvier 2022, consulté le 08 janvier 2022. URL : http://journals.openedition.org/germanica/7902 ; DOI : https://doi.org/10.4000/ germanica.7902 


\section{Émotion et évaluation : le cas des adjectifs affectifs dans les critiques de Marcel Reich-Ranicki}

Dominique Dias

ILCEA4, Université Grenoble Alpes

\section{Introduction}

À propos des œuvres des écrivaines qu'il admire, Marcel ReichRanicki affirme : « Nicht das Intellektuelle steht also im Vordergrund dieser Bücher, sondern das Emotionale, das Gemüt ${ }^{1} \gg$. Il souligne par-là l'importance émotionnelle qu'a l'écriture littéraire. L'expression de l'émotion est pourtant proscrite dans de nombreux genres discursifs car considérée comme trop subjective, irrationnelle et insuffisamment universelle. L'écriture journalistique est ainsi guidée par un principe de « délocutivité obligée ${ }^{2}$ » qui conduit le locuteur à effacer les marques de sa présence dans le texte. Pourtant dans certains genres spécifiques comme la critique littéraire, une prise de position est attendue et l'expression de l'émotion presque inévitable : cette coloration émotionnelle fait écho à celle de l'écriture littéraire. L'enjeu pour le recenseur est de passer d'une expérience individuelle du sujet à une expérience intersubjective donc communicable et incluse dans un processus évaluatif.

1. - Marcel Reich-Ranicki, Lauter Lobreden, München, DTV, 2000, p. 24.

2. - Patrick Charaudeau, « Discours journalistique et positionnements énonciatifs. Frontières et dérives », Semen, 22/2006, § 15. 
Nous proposons ici de revenir sur le lien entre évaluation et émotion en prenant pour objet d'étude les textes de Marcel Reich-Ranicki, connu pour son engagement émotionnel ${ }^{3}$ envers la littérature, en tant que lecteur et en tant que critique. À ce sujet, Thomas Anz ne peut s'empêcher de remarquer la récurrence d'un lexique des émotions dans ses critiques :

Reich-Ranickis Wirkungsargumente verwenden, soweit sie sich auf Unlustgefühle beim Lesen beziehen, mit Vorliebe Wörter wie « langweilen » oder « ermüden ». " Peinlich», « ärgerlich» oder «quälend» sind weitere Vokabeln der Abwertung, die er gerne benutzt ${ }^{4}$.

Parmi les différents lexèmes relevés par Thomas Anz, on trouve surtout des adjectifs dits affectifs, qui « énoncent, en même temps qu'une propriété de l'objet qu'ils déterminent, une réaction émotionnelle du sujet parlant en face de cet objet ${ }^{5} »$. On comprend que ces adjectifs affectifs sont tout désignés pour dire une expérience de la lecture et la communiquer au lecteur. Toutefois, on ne saurait se limiter à un simple relevé d'occurrences et à une approche strictement lexicographique. Si l'émotion participe de l'exercice attendu de l'évaluation, on sait par ailleurs que l'évaluation s'actualise à tous les niveaux (texte, langue et discours) et sous diverses formes (hyperstructure, modalité appréciative, configurations énonciatives) dans les recensions ${ }^{6}$. Il s'agit donc ici de déterminer le rôle des adjectifs affectifs dans l'évaluation, à la fois à l'échelle phrastique et à l'échelle textuelle. Le corpus considéré sera constitué des deux recueils Lauter Verrisse et Lauter Lobreden qui rassemblent un florilège de critiques assassines et de critiques élogieuses de Marcel ReichRanicki, deux positions extrêmes qui a priori laissent place à l'émotion. Du point de vue méthodologique, nous proposons une approche lexicale et sémantique, avec une dimension discursive afin de comprendre comment les adjectifs affectifs s'inscrivent dans l'appareil évaluatif.

Nous commencerons par présenter le corpus et quelques données générales recueillies avant de poser la question du rapport entre émotion et

3. - Cf. Klaus Eggensperger, "Para entender Marcel Reich-Ranicki », Pandaemonium, 23/2014, p. 139.

4. - Thomas Anz, « Zur Rationalität und Emotionalität literaturkritischer Kommunikation - am Beispiel von Marcel Reich-Ranicki », in : Heinrich Kaulen \& Christina Gansel (dir.), Literaturkritik heute: Tendenzen - Traditionen - Vermittlung, Göttingen, V\&R unipress, 2015, p. 17.

5. - Catherine Kerbrat-Orecchioni, L'énonciation : de la subjectivité dans le langage, Paris, Armand Colin, 2009, p. 94.

6. - Cf. Dominique Dias, « L'actualisation de l'évaluation et son effet structurant dans les critiques littéraires journalistiques », in : Thierry Gallèpe (dir.), Discours, texte et langue. La fabrique des formes et du sens, Frankfurt a.M., Peter Lang, 2016, p. 61-72. 
évaluation. Nous finirons par une étude de l'expression de la surprise pour observer la façon dont les affectifs structurent leur environnement textuel.

\section{Données générales du corpus}

Le corpus est composé des deux recueils qui constituent chacun un sous-corpus tant ils s'opposent dans leur visée discursive et leur forme. Dans Lauter Verrisse (1970), Reich-Ranicki compile et publie ses critiques à charge les plus emblématiques : il s'agit de 24 recensions au sens strict, c'est-à-dire des textes évaluatifs écrits à l'occasion de la parution d'un ouvrage littéraire. Dans Lauter Lobreden (1985), il s'agit à l'inverse de textes élogieux mais qui ne font pas la recension d'un ouvrage : ils sont parus à l'occasion de l'anniversaire d'un auteur ou de la remise d'une distinction, ils portent sur l'auteur et son œuvre en général. Dans les deux cas, les recueils ont permis d'inscrire dans la durée ces textes supposés éphémères en raison de leur publication dans la presse quotidienne ou hebdomadaire.

Pour le relevé des occurrences que nous présentons dans le tableau ci-dessous, nous avons pris en considération tous les adjectifs qui avaient le trait sémique affectif, cumulé ou non au trait sémique axiologique (catégorie a). Nous incluons les formes lexicalisées de participe I qui ne sont pas morphologiquement des adjectifs, mais qui occupent de manière privilégiée notamment les fonctions d'épithète et d'attribut. Comme définis précédemment, les adjectifs affectifs indiquent à la fois une propriété attribuée à l'objet considéré et une réaction émotionnelle. Si l'on reprend la typologie proposée par Catherine Kerbrat-Orecchioni ${ }^{7}$, ils appartiennent à la classe des adjectifs subjectifs, mais se distinguent des adjectifs dits axiologiques qui supposent quant à eux un jugement de valeur de la part du locuteur. Toutefois, certains adjectifs possèdent les deux propriétés et sont des axiologico-affectifs (catégories b et d) :

(1) Was sich hier abspielte, kommt mir widerwärtig und obszön vor: In Düsseldorf, der angeblich reichsten Stadt der reichen Bundesrepublik Deutschland, in einem Theaterneubau, der die öffentliche Hand nicht weniger als vierzig Millionen Mark gekostet hat und der sich zwischen dem Bürohaus eines der größten Konzerne Europas und $[\ldots]^{8}$.

(2) Freilich bietet das Buch ungleich mehr als sein schlichter Titel ankündigt. Es ist ein farbiges, streckenweise aufregendes Kapitel deutscher Kulturgeschichte mit vielen Paraphrasen über ein

7. - C. Kerbrat-Orecchioni, L'énonciation, op. cit., p. 94.

8. - Marcel Reich-Ranicki, Lauter Verrisse, München, DTV, 2008, p. 97. Sauf mention contraire, nous sommes à l'origine des soulignements. 
zentrales Thema: die Herrschenden und die Lehrenden, die Macht und der Geist ${ }^{9}$.

Dans la mesure où il s'agit ici d'étudier le rapport entre évaluation et émotion, nous avons par ailleurs exclu les affectifs (catégorie e) qui ne portaient pas sur l'ouvrage ou sur un élément permettant d'apprécier le travail de l'écrivain :

(3) Da gibt es Bürger der DDR mit widerlichen Zähnen und spinnenhaften Fingern, sie haben in ihrem Wesen ${ }^{10}[\ldots]$.

\begin{tabular}{|l|l|c|c|c|}
\hline & & Verrisse & Lobreden & Total \\
\hline (a) & Affectifs retenus & 95 & 33 & 128 \\
\hline (b) & $\begin{array}{l}\text { dont axiologiques } \\
\text { négatifs }\end{array}$ & 44 & 8 & 52 \\
\hline (c) & $\begin{array}{l}\text { dont axiologiques } \\
\text { positifs }\end{array}$ & 28 & 13 & 41 \\
\hline (d) & $\begin{array}{l}\text { dont exclusivement } \\
\text { affectifs }\end{array}$ & 23 & 12 & 35 \\
\hline (e) & Affectifs exclus & 7 & 9 & 16 \\
\hline
\end{tabular}

Tableau 1 : Relevé des occurrences

Parmi les 128 occurrences, on peut recenser 66 formes différentes. Si la répartition des axiologico-affectifs est dans l'ensemble à peu près équilibrée entre les deux pôles, on constate - conformément à l'attente que l'on pouvait avoir a priori - qu'elle se fait au profit des axiologiques négatifs dans Lauter Verrisse et au profit des axiologiques positifs dans Lauter Lobreden. Les adjectifs exclusivement affectifs - qui ne s'inscrivent donc pas sur un axe axiologique - sont en minorité et ils relèvent tous, comme nous le verrons plus loin, de l'expression de la surprise. Quant aux affectifs ne participant pas à l'évaluation de l'auteur ou de son travail, ils sont très minoritaires. Enfin, si l'on adopte une perspective thématique, on observe que les 66 formes différentes correspondent à une dizaine d'affects : nous entendons par là, à la fois des sentiments, des émotions ou des sensations. On dénombre par ordre de fréquence : la surprise (überraschend, erstaunlich, verblüffend), la gaieté (erfreulich, lustig, amüsant), l'horreur (schrecklich, schauerlich, abstoßend), l'émoi (rührend, bewegend), l'excitation (erregt, spannend,

9. - M. Reich-Ranicki, Lauter Lobreden, op. cit., p. 113.

10. - M. Reich-Ranicki, Lauter Verrisse, op. cit., p. 86. 
aufregend, ärgerlich), l'ennui (langweilig), la fatigue (ermüdend), l'étrangeté (komisch, merkwürdig), la déception (enttäuschend), la douleur (schmerzhaft). Les différentes formes constituent des variations d'intensité (überraschend < verblüffend) ou bien des variantes avec un trait axiologique (überraschend vs. erschreckend), voire se situent à la croisée entre deux affects (erschreckend $=$ surprise + horreur).

\section{L'émotion évaluative ou l'évaluation émotive?}

On admet généralement que les émotions participent d'une forme d'évaluation. En médecine sociale, les émotions sont conçues comme une réaction physique à un événement qui permettent de l'interpréter et de l'évaluer ${ }^{11}$. En sciences du langage, on les range parmi les systèmes de connaissance et d'évaluation qui servent à évaluer et à formuler un jugement sur les objets du monde ${ }^{12}$. Patrick Charaudeau ${ }^{13}$ affirme le lien inextricable qui existe entre émotions et système subjectif de valeurs, source d'évaluation. Par ailleurs, Iva Novakova et Julie Sorba ${ }^{14}$ montrent comment des noms d'émotions comme estime et déception contribuent à l'expression de l'évaluation au sein du discours journalistique.

Cette propriété qu'ont les affectifs d'être à la fois tournés vers un objet du monde et vers une source énonciative se reflète dans la structure argumentale que sous-tendent les procès qui sont associés à ces adjectifs (dérivés ou non de verbes). Autrement dit, les affectifs expriment la qualité d'un procès qui suppose un expérienceur, une source, voire une cause. La présence ou non en discours des différents rôles sémantiques attendus nous permet, à l'échelle phrastique, d'établir une typologie des emplois des affectifs.

Ainsi, dans cette recension d'un roman de Hans Erich Nossack, Marcel Reich-Ranicki explique qu'il n'a pas aimé le livre, mais que certains passages sont pourtant des morceaux de bravoure. L'apparente contradiction entre les deux jugements n'a, d'après lui, rien de surprenant :

(4) Beides kommt übrigens für diejenigen, die Hans Erich Nossacks sonderbare Sicht und hartnäckige Eigenart kennen, keineswegs

11. - Cf. Thomas Hülshoff, Emotionen, München, Ernst Reinhardt Verlag, 2012.

12. - Cf. Monika Schwarz-Friesel, Sprache und Emotion, Tübingen, A. Francke, 2013.

13. - Patrick Charaudeau, «Une problématisation discursive de l'émotion. À propos des effets de pathémisation à la télévision », in : Christian Plantin, Marianne Doury \& Véronique Traverso (dir.), Les émotions dans les interactions, Lyon, Presses Universitaires de Lyon, 2000, p. 130.

14. - Iva Novakova et Julie Sorba, «L'évaluation à travers les émotions : le cas d'estime et de déception », Langue française, 184/2014, p. 75-91. 
überraschend: Nicht die Existenz der Schwächen und Vorzüge ist hier also verwunderlich, wohl aber ihre Intensität ${ }^{15}$.

Ici l'affectif überraschend est entouré de la source (les deux jugements pronominalisés par beides) et de l'expérienceur (le connaisseur de l'œuvre de Nossack). La réalisation de ces deux rôles sémantiques pourtant inhérents au sémantisme des affectifs est un cas assez peu représenté dans le corpus (voir également exemple 1). Notamment le rôle de l'expérienceur est peu présent à moins d'en faire, comme ici, un lecteur averti, en connivence avec le recenseur.

Dans l'immense majorité des cas, c'est surtout la source qui est mentionnée au détriment de l'expérienceur, comme dans cette recension d'un livre d'Adolf Muschg :

(5) Mehrere Personen, darunter zwei Juristen und ein Psychologe, gehen diesen Fragen geduldig nach, und was sie schließlich erfahren - das meiste stammt von dem verwundeten Zerutt, der freilich nur widerwillig und recht umständlich Auskunft erteilt -, füllt zwar die fast 400 Seiten des Romans, scheint aber dennoch dürftig und enttäuschend ${ }^{16}$.

L'affectif enttäuschend correspond au procès enttäuschen qui suppose à la fois en termes de rôles sémantiques, une source et un expérienceur. Or, dans cet exemple, si le contenu du roman est la source de la déception, l'expérienceur n'est en revanche pas explicité : dans le cadre du genre recension, on peut en déduire qu'il s'agit du recenseur et/ou de tout lecteur potentiel. Cette configuration est assez représentative des différentes occurrences du corpus : contrairement aux verbes affectifs, les adjectifs affectifs présentent la commode particularité de ne pas nécessiter la réalisation en discours de l'expérienceur, qui est pourtant un rôle sémantique latent. Cet effacement de l'expérienceur présente plusieurs avantages. Comme nous l'avons suggéré, sa mention n'est pas pertinente dans une scène générique aussi balisée. Peu de référents peuvent potentiellement jouer ce rôle : $\mathrm{S}$ ist [für mich/den Leser] $\mathrm{A}_{\mathrm{aff}}$ Le fait de mentionner l'expérienceur tendrait à souligner le caractère subjectif de l'affectif et restreindrait la portée de l'évaluation. L'absence de l'expérienceur en discours répond en partie à la contrainte de l'effacement énonciatif qui tend à rendre le propos plus neutre en apparence et plus universel. Cela permet par ailleurs, en discours, un traitement similaire des affectifs et des axiologiques. Effectivement, si l'expérienceur n'est jamais mentionné, en revanche, la source de l'émotion apparaît quasi systématiquement : en termes syntaxiques elle occupe alors la

15. - M. Reich-Ranicki, Lauter Verrisse, op. cit., p. 50.

16. — Ibid., p. 157. 
fonction sujet (et l'affectif est alors attribut du sujet, avec une prédication première) ou bien elle est base d'un groupe nominal dont l'affectif est l'épithète (avec une prédication seconde). On peut schématiser ces deux cas de figure comme suit, où $\mathrm{S}$ est la source et $\mathrm{A}_{\text {aff }}$ est un affectif :

$$
\begin{gathered}
\mathrm{S}=\mathrm{A}_{\mathrm{aff}} \\
\mathrm{GN}\left(\mathrm{A}_{\mathrm{aff}} \mathrm{S}\right)
\end{gathered}
$$

Dans les deux cas de figure, on assiste à la macro-opération de l'aspectualisation qui se décompose en deux opérations : la première est celle de la fragmentation ${ }^{17}$ qui consiste à sélectionner des parties de l'objet considéré. Autrement dit : S n'est pas toujours le même objet, cela peut être l'ouvrage, le style, le travail de l'auteur, un chapitre... La seconde opération est celle de la qualification qui consiste en une « mise en évidence de propriétés du tout et/ou des parties sélectionnées par l'opération de fragmentation. L'opération de qualification est le plus souvent réalisée par la structure du groupe nominal nom + adjectif et par le recours prédicatif au verbe être ${ }^{18} \gg$. En ce sens, on ne distingue pas les affectifs des axiologiques puisque dans les recensions, ils participent d'opérations descriptives similaires.

Enfin, le troisième et dernier cas de figure cumule l'effacement de l'expérienceur et de la source. Cela peut être dû au fait que la source n'est pas un élément issu de l'opération de fragmentation. Ainsi, à propos de l'écrivaine Anna Seghers, Marcel Reich-Ranicki écrit :

(6) Schon konnte man hoffen, es sei ihr gelungen, den schauerlichen Tiefpunkt ihrer schriftstellerischen Laufbahn [...] zu überwinden ${ }^{19}$.

Dans la structure GN ( $\left.\mathrm{A}_{\mathrm{aff}} \mathrm{S}\right)$, telle que nous l'avons décrite, $\mathrm{S}$ ne correspond pas ici à un élément du roman ou du travail de l'écrivain. L'adjectif par son sens axiologique dénote une évaluation, mais son sens affectif consiste avant tout en une intensification du sémantisme du substantif. Il en va de même pour les affectifs employés en fonction d'adjectifs d'adjectifs. Les deux rôles sémantiques inhérents au sémantisme de ces adjectifs n'étant pas réalisés, on comprend que leur emploi s'assimile alors davantage à celui des intensifieurs :

17. - La fragmentation ou partition consiste en une « sélection de parties de l'objet de la description », in : Jean-Michel Adam, La linguistique textuelle, Paris, Armand Colin, 2015, p. 174.

18. - Ibid.

19. - M. Reich-Ranicki, Lauter Verrisse, op. cit., p. 46. 
(7) Das Buch scheint reichhaltig zu sein. Weshalb ist es doch erschreckend armselig ${ }^{20}$ ?

Dans ce cas, les affectifs participent de manière indirecte à l'évaluation, en intensifiant le sens d'autres adjectifs en général axiologiques, comme ici armselig, qui cristallisent l'évaluation.

\section{L'expression de la surprise}

Comme nous l'avons évoqué plus haut, l'évaluation est une activité qui a lieu à plusieurs niveaux. Pour comprendre quelle place occupent les affectifs dans le système évaluatif, nous proposons d'étudier de plus près le cas de la surprise. Le choix de cet affect a été fait pour plusieurs raisons. Il s'agit tout d'abord de l'affect le plus fréquent dans le corpus (32 occurrences) et il présente donc, contrairement à d'autres affects, assez de matériau pour une analyse plus fine. Par ailleurs, il s'agit dans le corpus du seul cas d'affectif non axiologique en langue. La surprise est d'ailleurs présente dans les deux sous-corpus. La façon dont elle participe de l'évaluation est d'autant plus intéressante. Enfin, elle s'exprime au moyen de plusieurs adjectifs affectifs, en l'occurrence : überraschend, verwunderlich, verblüffend, erstaunlich et erschreckend, cette dernière forme étant la seule à être axiologique.

L'objectif de cette étude de cas n'est pas de reprendre les analyses en termes de rôles sémantiques qui ont pu être faites précédemment, mais d'observer en quoi et à quelle échelle le sémantisme des adjectifs affectifs exprimant la surprise est susceptible de déterminer leur environnement textuel.

Ainsi, l'une des premières configurations récurrentes est celle de groupes prépositionnels souvent compléments circonstanciels de manière ou attributs. Tel est le genre d'associations lexicales que les affectifs de la surprise sont susceptibles de déterminer à l'échelle phrastique :

(8) Besonders fällt in dem Roman eine Verbindung auf, die mir in der Tat außergewöhnlich scheint - von verblüffender Direktheit nämlich mit gehobener Umständlichkeit ${ }^{21}$.

(9) Rebellierende Schüler, Wehrdienstverweigerer, eine linke Lehrlingsgruppe, die eine Wohngemeinschaft bildet, ein Schulungslager der Spartacus-Leute - daraus werden bei Muschg mit verblüffender Regelmäßigkeit epische Schnörkel und Arabesken ${ }^{22}$. 
(10) Schleierhaft bleibt, warum man bei solchen Überlegungen mit erstaunlicher Regelmäßigkeit jene ausspart, an die man sich wohl ebenfalls halten könnte - nämlich die Autoren ${ }^{23}$.

(11) Im Gegenteil: Dieser Stil ist von verblüffender Unmittelbarkeit und Frische und vereint in so hohem Maße Anschaulichkeit mit Musikalität, daß es uns leicht fällt, über gelegentliche Schwächen des Buches, zumal in seinem Mittelteil, hinwegzusehen ${ }^{24}$.

On constate une certaine récurrence dans les lexèmes associés désignant le caractère direct (Direktheit, Unmittelbarkeit) ou la régularité (Regelmäßigkeit). Au sein de notre corpus, c'est le lexème verblüffend, défini comme plus intense que erstaunlich ou überraschend, qui sert en général à former ces locutions récurrentes. Le seul autre cas de spécialisation d'un lexème de la surprise dans une configuration spécifique est celle de l'axiologico-affectif erschreckend que l'on trouve dans le corpus presque exclusivement en fonction d'adjectif d'adjectif, une fonction que nous avons associée à un rôle d'intensifieur. Étant donné son sens axiologique négatif, il porte uniquement sur d'autres axiologiques négatifs (erschreckend oberflächlich, erschreckend armselig).

Outre ces formes de locutions évaluatives, ce qui nous semble particulièrement intéressant est le caractère textogène ${ }^{25}$ des affectifs exprimant la surprise. Effectivement, force est de constater que la portée de ces adjectifs dépasse le cadre intraprédicatif de la phrase, qu'il s'agisse d'une prédication seconde (l'adjectif servant d'épithète à un substantif) ou d'une prédication première (l'adjectif ayant la fonction d'attribut du sujet). Dans les occurrences ci-dessous, on observe ainsi un rapport de coréférence entre les unités GN $\left(\mathrm{A}_{\mathrm{aff}} \mathrm{S}\right)$ et les énoncés situés au-delà des deux points :

(12) Für die hier so reichlich gebotenen Tricks und Gags, Schnitte und Sprünge, Rückblenden und Überblenden findet Grass eine im ersten Augenblick verblüffende formale Rechtfertigung: Starusch sieht seine Vergangenheit und seine Phantasien auf einem neben dem zahnärtzlichen Behandlungssessel zwecks Ablenkung des Patienten aufgestellten Fernseh-Bildschirm ${ }^{26}$.

23. - Ibid., p. 161.

24. - M. Reich-Ranicki, Lauter Lobreden, op. cit., p. 170.

25. - Au sens que lui donnent I. Novakova et J. Sorba, à savoir capable de structurer l'environnement textuel. Cf. Iva Novakova, Julie Sorba, « Les adjectifs d'affect : complexité sémantique et profils discursifs ", in : Franck Neveu/Peter Blumenthal (dir.), Actes du 4e Congrès mondial de linguistique française, Les Ulis, EDP Sciences, 2014, p. 640.

26. - M. Reich-Ranicki, Lauter Verrisse, op. cit., p. 116. 
(13) In einem ihrer späten Gedichte findet sich die überraschende Zeile: «Alt sein heißt suchen. » Bis zum Ende blieb Marie Luise Kaschnitz dieser Devise treu ${ }^{27}$.

Avec l'autre schéma $\mathrm{S}=\mathrm{A}_{\text {aff }}$, on observe le même rapport de coréférence entre $S$ et l'énoncé suivant. L'opération d'aspectualisation réalisée par $\mathrm{A}_{\mathrm{aff}}$ est donc valable à la fois pour $\mathrm{S}$ et pour l'énoncé coréférent :

(14) Was dies hier bewirkt, scheint mir einigermaßen erschreckend: Ein Schriftsteller, an dessen Begabung ich glaube und an dessen Aufrichtigkeit überhaupt nicht gezweifelt werden kann, erzählt, was er selbst erlitten hat und was dennoch aus zweiter Hand stammt ${ }^{28}$.

Dans tous les cas, l'affect surprise est étendu à son environnement textuel et très souvent, cet environnement textuel est constitué par un énoncé représentant le contenu littéraire évalué. Autrement dit, il s'agit d'un énoncé dont la prise en charge énonciative est réalisée par un autre énonciateur, soit sous la forme d'une citation (13), soit sous la forme d'un résumé descriptif (12). Les deux points revêtent ici une fonction d'opérateur métatextuel assurant la liaison entre l'évaluation affective et le texte évalué. Il s'agit là d'un cas de surénonciation ${ }^{29}$ dans lequel l'énonciateur-recenseur adopte une position surplombant celle de l'énonciateur-écrivain. Cette configuration énonciative est typique des recensions $^{30}$ dans la mesure où elle traduit le rapport hiérarchique existant entre le recenseur et le texte source qu'il évalue.

La surprise étant un affect $a$ priori non axiologique, il est propice à créer une attente chez le lecteur. Ces lexies revêtent un caractère textogène dans la mesure où dans l'environnement textuel qui les suit, on attend que cet affect se charge d'un signifié axiologique. La surprise peut se charger d'un signifié négatif, elle est alors causée par le caractère invraisemblable d'un roman ou bien elle est la marque d'une incompréhension qui laisse le lecteur perplexe et appelle un décodage de la part du recenseur. Ainsi la surprise peut être un indice d'invraisemblance comme dans le cas de ce personnage d'un roman de Stefan Andres :

(15) Das resolute Mädchen, das häufig ihre Familienangehörigen belauschen muß, zeichnet sich bisweilen durch eine verblüffende Reife aus - und bei anderen Gelegenheiten durch überraschende Kindlichkeit. Den Leser, der etwa meinen sollte, beides sei ihrem Alter nicht eben angemessen, beruhigt Stefan Andres, indem er

27. - M. Reich-Ranicki, Lauter Lobreden, op. cit., p. 50.

28. - M. Reich-Ranicki, Lauter Verrisse, op. cit., p. 135.

29. - Cf. Alain Rabatel, «L'effacement énonciatif dans les discours rapportés et ses effets pragmatiques », Langages 38/2004, p. 8.

30. - D. Dias, « L'actualisation... », op. cit., p. 69. 
Vater Odilo die außergewöhnlichen Möglichkeiten der Tochter wahrnehmen lässt: «Welch eine Mischung, diese Felizitas! In manchen Augenblicken kommt sie mir wie fünfzehn, manchmal wie fünf vor... $»^{31}$.

La surprise sert de déclencheur argumentatif et textuel : en l'espèce, la surprise est marquée par deux affectifs, suivis par la mise en scène des interrogations que peut légitimement se poser le lecteur et par la réponse apportée par l'auteur. Le recenseur orchestre ainsi un dialogue in absentia entre son évaluation, la voix fictive du lecteur et la réponse de l'auteur. C'est bien le travail de recension qui est ici mis en scène et qui légitime en même temps l'évaluation du recenseur.

Effectivement, l'expression de la surprise permet au recenseur de légitimer son rôle d'expert de l'écriture littéraire, par sa culture littéraire donnée à entendre dans les développements explicatifs programmés par les affectifs. Ainsi, dans cette recension d'un roman de Günter Herburger, Marcel Reich-Ranicki qualifie d'étonnantes les anamnèses et les prolepses employées par l'auteur :

(16) In der « Messe » wimmelt es von plötzlichen Zeitsprüngen und überraschenden Rückblenden und Antizipationen, die nur bisweilen einigermaßen motiviert sind, von allerlei Montagen und Verschachtelungen, die meist nicht viel ergeben ${ }^{32}$.

Le paragraphe suivant revient sur la composition du roman et confère au recenseur une posture d'expert de la littérature, où l'expression de la surprise possède une capacité structurante. Même si la surprise n'est pas, en soi, axiologiquement marquée, dans les recensions, le contexte la charge d'une connotation positive ou négative.

La surprise peut, en effet, être vue comme un affect positif. Le lecteur exige d'être surpris, l'absence de surprise est alors synonyme d'ennui et de prévisibilité. Ainsi à propos de la carrière de Horst Krüger qui a notamment officié en tant que journaliste culturel, Reich-Ranicki souligne le rapprochement, qu'il qualifie d'« étonnant», que l'on peut faire entre la critique littéraire et d'autres formes littéraires :

(17) Dies aber rückt das Feuilleton, wie es Krüger zu üben weiß, in die Nähe nicht etwa anderer Prosaformen, sondern - sehr überraschend - in die unmittelbare Nachbarschaft der Lyrik. Der Lyriker zeigt seine Epoche und seine Welt, indem er sich zeigt und beschreibt. Nicht anders verfährt der Feuilletonist Horst Krüger:

31. - M. Reich-Ranicki, Lauter Verrisse, op. cit., p. 58.

32. - Ibid., p. 145. 
Er behandelt sich selber als eine Art Versuchsperson, die er den Wirkungen seiner Zeit, unserer Zeit, aussetzt ${ }^{33}$.

Là encore, la surprise, qu'elle soit négative ou comme ici positive, légitime une séquence explicative. Le paragraphe suivant développe ainsi un parallèle entre le travail de critique et celui d'écrivain. Sans l'expression de la surprise, cette digression explicative pourrait être perçue comme superflue. Si nous avons choisi ici de présenter l'expression de la surprise en raison de sa très haute fréquence dans les recensions de Marcel Reich-Ranicki, il convient de préciser que tous les affects ne sont pas pareillement textogènes ${ }^{34}$. C'est d'ailleurs peut-être justement le caractère profondément textogène de la surprise qui explique en retour sa grande fréquence.

\section{Conclusion}

Nous sommes partis du constat fait par certains de la grande fréquence de lexies relevant de l'affect dans les recensions de Marcel Reich-Ranicki pour nous interroger sur leur rôle dans l'évaluation. Un premier relevé a montré effectivement la présence de nombreux adjectifs affectifs que l'on peut classer en une dizaine d'affects. Mais si les affectifs se distinguent des axiologiques par les rôles sémantiques qu'ils impliquent, force est de constater que dans les recensions, la place de l'expérienceur, voire de la source tend à s'effacer. Autrement dit, les affectifs connaissent un emploi qui les rapproche des axiologiques, répondant par là en partie aux exigences de l'effacement énonciatif de l'écriture journalistique. Plus déterminant en revanche est le rôle que joue le sémantisme des affectifs dans la structuration de leur environnement textuel. Le fait que l'affect ne soit pas en soi axiologiquement marqué permet de créer une attente et de développer un scénario qui dans le cas de la surprise n'est en rien prédéterminé. La question qui reste ouverte est celle du rapport entre adjectifs affectifs et expressivité. Catherine Kerbrat-Orecchioni rappelle notamment comment Bally conçoit la langue en opposition à l'expressivité : «plus un signe est chargé d'affectivité, et moins il est véritablement linguistique ${ }^{35} \gg$, c'est-à-dire que l'expressivité lutte contre les deux propriétés des signes linguistiques, à savoir l'arbitrarité et la linéarité. Or, les observations que nous avons pu mener au sujet des

33. - M. Reich-Ranicki, Lauter Lobreden, op. cit., p. 93.

34. - Les adjectifs affectifs n'ont pas tous le même profil discursif, certains engendrant un scénario discursif plus dépouillé que d'autres. Cf. Iva Novakova, Julie Sorba, « Les adjectifs d'affect... », op. cit., p. 646.

35. - Catherine Kerbrat-Orecchioni, " Quelle place pour les émotions dans la linguistique du XXe siècle ? Remarques et aperçus », in : C. Plantin, M. Doury \& V. Traverso (dir.), Les émotions, op. cit., p. 36. 
adjectifs affectifs dans les recensions de Marcel Reich-Ranicki montrent comment ils sont au contraire proprement linguistiques, comment ils structurent leur environnement textuel et comment ils mettent le recenseur dans une posture de surénonciateur. En d'autres termes, il serait trompeur de voir dans le recours à des lexèmes dénotant des sentiments la marque d'une expressivité spontanée et irrationnelle. En reconstituant l'organisation autour des affectifs, on accède au contraire à la structuration des stratégies évaluatives susceptibles de transmettre une expérience intersubjective. 
